\title{
Meeting the Challenge of COVID-19 Pandemic in Mental Health Care in a Tertiary-Care General Hospital in India
}

\author{
Mamta Sood ${ }^{1} \quad$ Rakesh K. Chadda ${ }^{1}$ \\ 1Department of Psychiatry, All India Institute of Medical Sciences, \\ Ansari Nagar, New Delhi, India
}

Address for correspondence Mamta Sood, MD, Department of Psychiatry, All India Institute of Medical Sciences, Ansari Nagar, New Delhi110029, India (e-mail: soodmamta@gmail.com).

\begin{abstract}
Keywords

- COVID-19

- functioning

- India

- medical college

- psychiatry

Medical profession has proved its versatility in the adoption of many strategies to deal with the impact of COVID-19. All India Institute of Medical Sciences (AIIMS), New Delhi, India is a large public funded medical school and general hospital in North India. Psychiatry department is one of the 42 departments at AIIMS. COVID-19 has impacted all the functions of the department: clinical care, teaching and training, research and other roles. In this communication, we report the strategies, simple yet effective improvisations to meet the challenges of COVID-19 so as to continue the basic functions of the discipline. This could serve as a model for continued functioning of a tertiary care teaching medical facility in a pandemic situation.
\end{abstract}

\section{Introduction}

The COVID-19 pandemic is an extraordinary event in the history of health care systems and has shaken up the very foundation on which these systems operate. However, the medical profession has proved its versatility in the adoption of many strategies to deal with its impact. In India, an almost complete nationwide lockdown was imposed on March 24, 2020, which remained till May 31, 2020, and it impacted every facet of life. ${ }^{1}$

The All India Institute of Medical Sciences (AIIMS), New Delhi, India, is a large, public-funded medical school and general hospital in North India. The Department of Psychiatry is one of the 42 departments at AIIMS. ${ }^{2}$ The lockdown for dealing with COVID-19 impacted its functions: clinical care, teaching and training, and research. The outpatient services were closed on March 25, 2020.

In this communication, we report the simple yet effective improvisations to meet the challenges of COVID-19 so as to continue the basic functions of the psychiatry discipline in the beginning and during the lockdown period when there was lot of uncertainty. In India, there are 542 medical colleges teaching $\mathrm{MBBS}^{3}$ and presence of psychiatry department is mandatory in all the medical colleges. ${ }^{4}$ The mode of strategies used by us could serve as a model for continued functioning in a tertiary-care teaching medical facility in a pandemic situation.

\section{Clinical Services}

The Department of Psychiatry runs daily outpatient services, seven specialty clinics, community services at two centers, consultation-liaison (CL) services, and emergency care integrated with hospital emergency services. Annually, on average, it provides services to about 80,000 persons in outpatient and 10,000 persons in community clinics, 1,200 persons in CL services, and 1,000 persons in the main hospital emergency. Every year, about 300 inpatients are admitted in the published online June 9, 2021
DOI https://doi.org/ $10.1055 / \mathrm{s}-0041-1729774$ ISSN 0379-038X
(C) 2021. National Academy of Medical Sciences (India).

This is an open access article published by Thieme under the terms of the Creative Commons Attribution-NonDerivative-NonCommercial-License, permitting copying and reproduction so long as the original work is given appropriate credit. Contents may not be used for commercial purposes, or adapted, remixed, transformed or built upon. (https://creativecommons.org/licenses/by-nc-nd/4.0/). Thieme Medical and Scientific Publishers Pvt. Ltd. A-12, 2nd Floor, Sector 2, Noida-201301 UP, India 
psychiatry ward (a 32-bedded facility). ${ }^{2}$ COVID-19 impacted all the clinical services. To continue clinical care, the following steps were taken.

Outpatient Services: These services were closed on March 25, 2020. In sync with the Ministry of Health and Family Welfare Telemedicine Practice Guidelines, ${ }^{5}$ telepsychiatry services were started on March 31, 2020, with the help of the institute's computer facility, for patients already enrolled. Mobile phones with internet facility were used and audio call, WhatsApp, and general text messaging functions were used. Two senior residents (Post MD) and two junior residents (MD trainees) under the supervision of three faculty (Post MD of more than 3 years) provided telepsychiatry services. Till May 31, 2020, about 2,500 persons were provided consultation by teleconsultation team.

Inpatient Services: The psychiatry ward, located in the main building of the hospital, was declared non-COVID area. Out of 32 beds, 24 beds were reserved for surgical trauma emergency as the hospital's trauma center was converted to COVID hospital. All categories of health care personnel (HCP) underwent training and sensitization sessions, arranged by the hospital administration department. Discharge of stable patients was expedited; group activities were suspended and routine admissions were stopped. Only psychiatric patients with emergency problems were admitted. Psychiatry ward is an open ward with shared washrooms and spaces, and patients are admitted with family members. In a functional isolation room with attached bathroom, every new psychiatric admission was kept for the first 5 days. All ward surfaces were cleaned at regular periods with $1 \%$ sodium hypochlorite, every 2 to 4 hours. All patients, attendants, staff, and visitors were checked using COVID-19 symptom checklist daily; they were asked to wear masks and practice hand hygiene. For contact tracing in event of a positive case, a list of all the staff and personnel working at the ward was made. All inhabitants of the ward were advised not to have meals or coffee/tea together. The set of guidelines on the use of personal protection equipment (PPE) for HCP was continually updated as per national and international recommendations. Initially, triple-layer mask was recommended that was upgraded to N95 mask. Later, all of the HCP wore level I PPE (N95 mask, gown, gloves, and eye goggles). Donning and doffing areas were identified and prepared as per the protocol. All relevant information was displayed on the posters. Till May 31, 2020, the ward operated at the allocated capacity ( 6 beds) and a total of 14 patients were admitted.

Emergency services: A junior and a senior resident under the supervision of faculty regularly attended the psychiatric emergencies reporting in the main hospital casualty. To minimize the risk of COVID-19, in-person consultations were minimized. Usually, a phone call with physician on call in emergency asking for detailed history and other information and talking with patient sufficed. Instructions were given for referral to other departments, investigations, or admission. Sometimes, video call through WhatsApp was also used to examine the patient. If there was a need for in-person consultation, PPE was used with donning and doffing done in the designated area in the emergency. If there was suspicion of COVID-19, opinion from the infectious diseases team was also sought. Till May 31, 2020, 150 persons were seen in emergency services.

CL services: The CL psychiatry team received regular calls from other departments of hospital. The strategies used were same as for emergency services. These were, in order of use, phone, video, and in-person consultation, depending on the need and available resources.

Psychiatric care in COVID areas of the hospital: $50 \%$ of residents and $15 \%$ of faculty were posted for general medical duty in the COVID areas of the hospital. They also provided psychiatric consultation to persons needing consultation in these areas.

\section{Teaching and Training}

The academic activities in the department include teaching and training medical and nursing undergraduates, postgraduates in psychiatry, postgraduates from other departments on clinical rotation in psychiatry, and $\mathrm{PhD}$ scholars in clinical psychology. Theory lectures were shifted to a central online platform called Student Advanced Resources and Learning (SARAL) platform, ${ }^{6}$ designed and run by the institute's computer facility. Clinical postings were also shifted to online mode. For the postgraduates, there are weekly seminars, journal club, case conference, thesis protocol, and midterm thesis presentations. For the rest, initially the academics were hosted on campus SET (Skills, e-Learning, Telemedicine) facility. Due to the risks associated with in-person instruction at SET facility, academic teaching was shifted to online platforms. Within 2 weeks, on April 11, 2020, the first academic program was started. Since then, the department has conducted all the programs regularly with good attendance and participation. For postgraduates, theory and practical examinations take place twice a year-May and December. The postgraduate examinations were conducted in June 2020 as they were to end their term of 3 years in June. Theory papers were conducted in the institute's examination section with social distancing and in two time slots ( 9 a.m. -12 p.m. and 2-5 p.m.). Scanned copies of theory papers were sent to the examiners. The practical exam was conducted maintaining protocols of social distancing, wearing a mask, and hand hygiene. There were no patients for the practical examination. The format for examination remained the same: long and short psychiatry case, neurology case, and viva. For the cases, case histories and case vignette were given to the candidates. One of the external examiners was in a different city and took it online. We had also conducted mock practical examinations for the exam-going postgraduates so that they get acquainted with the new pattern. In December 2020, the external examiners joined online, though real-life cases were used.

\section{Research}

The department has been involved in interdepartmental, collaborative, and departmental research at the institute level. Several national and international agencies fund the research activities. In a year, we publish about 150 papers and book 
chapters. To decongest the hospital spaces, the research staff worked remotely. No new patients/subjects were recruited for research purposes and phone follow-ups with already recruited patients/subjects were conducted. Backend activities related to the projects such as data analysis and report writing were carried out. As MD students were not able to recruit subjects for their theses (a mandatory requirement), suitable modifications were done in the protocols and submission dates were postponed, so that their deadlines for the examinations could be completed.

\section{Information, Education, and Communication Activities}

The COVID-19 pandemic is associated with psychosocial stress reactions among the general public as well as the health care workers. WHO posters ${ }^{7}$ on "Coping with stress during the 2019-nCoV outbreak" and "Helping children cope with stress during 2019-nCov outbreak" were translated into 11 Indian languages after taking due permission from the WHO. Educational video clips for general public, children and adolescents, single men, single women, and elderly population were developed. Guidance notes were developed for health care workers in English and Hindi to identify stress in themselves and to cope with it. Guidance notes for general population for dealing with mental health issues in persons quarantined or put in isolation, physicians dealing with patients/suspects with COVID-19, and mental health professionals were also developed. These were widely circulated and displayed at the institute's website. ${ }^{8}$ In the psychiatry ward, posters in simple language were displayed for educating about the preventive measures and donning and doffing procedure for COVID-19.

\section{Conclusion}

This article provides evidence that something can always be done in times of crisis. It is pertinent to mention that several compromises had to be made in various aspects of functioning. AIIMS, being a premiere teaching institution of the country, is well equipped in terms of staff, equipment, and funds. Other teaching institutions may have found the situation more challenging because of limited infrastructure. Nevertheless, strategies used have been introduced in this article, which can be used at different places with suitable modifications according to the local resources and requirements.

Future direction: The experiences shared in this article can be of help in developing suitable policy to be used in similar situations emerging in future. The Department of Psychiatry adapted to the impact of COVID-19 and lockdown by adopting simple strategies. Some of these strategies and lessons learnt are going to remain with us even after this pandemic is over. Telepsychiatry, especially for follow-up patients, and online platforms for teaching and meetings are here to stay.

\section{Author Contributions}

M.S. and R.K.C. conceptualized the manuscript. M.S. wrote the first draft of the manuscript. R.K.C. added more content to the first draft. Both the authors edited and prepared the final draft.

\section{Conflict of Interest}

None declared.

\section{References}

1 Ministry of Health and Family Welfare. Government of India. COVID 19 India. Available at: https://www.mohfw.gov.in/. Accessed June 25, 2020

2 All India Institute of Medical Sciences. 63rd Annual Report 2018-2019. Available at: https://www.aiims.edu/ images/pdf/annual_reports/annual\%20report19-e-20-1-20. pdf. Accessed June 4, 2020

3 Medical Council of India. List of college teaching MBBS. Available at: https://www.mciindia.org/CMS/information-desk/for-students-to-study-in-india/list-of-collegeteaching-mbbs. Accessed July 29, 2020

4 Medical Council of India. Minimum standard requirements for the medical college. Available at: https://www.mciindia. org/documents/informationDesk/STANDARD-FOR-250.pdf. Accessed July 29, 2020

5 Telemedicine practice guidelines. Enabling registered medical practitioners to provide healthcare using telemedicine. Available at: https://www.mohfw.gov.in/pdf/Telemedicine. pdf. Accessed June 10, 2020

6 AIIMS Saral (Student Advanced Resources and Learning Platform). Available at: https://saral.aiims.edu/. Accessed June 3, 2020

7 World Health Organization. How to cope with stress during 2019-nCoV outbreak. Available at: https://www.who. int/emergencies/diseases/novel-coronavirus-2019/advice-forpublic. Accessed June 7, 2020

8 AIIMS Covid Portal. Available at: https://covid.aiims.edu/ information-for-public/. Accessed June 3, 2020 